\title{
A NOTE ON THE CONSTANT THERMAL CONDUCTIVITY HYPOTHESIS AND ITS CONSEQUENCE FOR CONDUCTION HEAT TRANSFER PROBLEMS
}

\author{
R. M. S. Gama \\ Universidade do Estado do Rio de Janeiro \\ Faculdade de Engenharia \\ Departamento de Engenharia Mecânica \\ Rua São Francisco Xavier 524 sala 5020A \\ CEP 20550-013, Rio de Janeiro, RJ, Brasil \\ rsgama@terra.com.br \\ Received: October 29, 2014 \\ Revised: November 30, 2014 \\ Accepted: December 30, 2014
}

\section{ABSTRACT}

This work discuss the usual constant conductivity assumption and its consequences when a given material presents a strong dependence between the temperature and the thermal conductivity. The discussion is carried out considering a sphere of silicon with a given heat generation concentrated in a vicinity of its centre, giving rise to high temperature gradients. This particular case is enough to show that the constant thermal conductivity hypothesis may give rise to very large errors and must be avoided. In order to surpass the mathematical complexity, the Kirchhoff transformation is used for constructing the solution of the problem. In addition, an equation correlating thermal conductivity and the temperature is proposed.

Keywords: conduction heat transfer, Kirchhoff transformation, thermal conductivity

\section{NOMENCLATURE}

div denotes the divergence

grad denotes the gradient

$h$ convection coefficient

$k \quad$ thermal conductivity

n the unit outward normal

$\dot{q} \quad$ internal supply (per unit volume)

$Q \quad$ heat dissipation

$R_{2} \quad$ radius of the sphere

$R_{1} \quad$ radius of the region with $\dot{q}>0$

$r$ radial variable

$T$ absolute temperature

$T_{\infty} \quad$ temperature of the environment

$x, y, z$ usual Cartesian coordinates

\section{Symbols}

$\mathrm{K}[T]$ the Kirchhoff transformation

$K^{-1}[\omega]$ the inverse of $K$

$\omega \quad$ the function arising from $\mathrm{K}[T]$

$\Omega \quad$ bounded open set

$\partial \Omega \quad$ the boundary of $\Omega$

\section{INTRODUCTION}

The thermal conductivity depends on the temperature for all the materials. Nevertheless, conduction heat transfer problems are, in general, simplified by means of the constant thermal conductivity assumption (Incropera and Dewitt, 1996).
The main objective of this work is to show that there exist situations in which the thermal conductivity assumption gives rise to non negligible errors. In such cases, the dependence between the thermal conductivity $k$ and the temperature $T$ must be taken into account.

Aiming to illustrate the importance of taking into account the dependence between $k$ and $T$, let us consider the high purity silicon and its thermal conductivity. In general, the thermal conductivity for this material is assumed to be $130 \mathrm{~W} / \mathrm{mK}$ (Goldberg et al., 2001). But this value corresponds, approximately, to the conductivity at $340 \mathrm{~K}$. Table 1 below (Glassbrenner et al., 1964) shows that the thermal conductivity of the silicon is strongly dependent on the temperature.

Table 1 . The thermal conductivity $k$ for the silicon.

\begin{tabular}{|c|c|c|c|c|}
\hline$T(K)$ & $k(W / m K)$ & \& & $T(K)$ & $k(W / m K)$ \\
\hline 200 & 264 & $?$ & 500 & 76.2 \\
\hline 250 & 191 & $?$ & 600 & 61.9 \\
\hline 300 & 148 & $?$ & 800 & 42.2 \\
\hline 350 & 119 & $?$ & 1000 & 31.2 \\
\hline 400 & 98.9 & $\mathrm{~s}$ & 1200 & 25.7 \\
\hline
\end{tabular}

An approximation for the above table, within the range $200 K<T<1200 K$, is proposed here by means of the following equation

$$
k=\hat{k}(T)=\left\{\frac{1220}{\sqrt{T}(\ln T)}\right\}^{2}
$$

in which the error is less than 5\%. Equation (1) gives rise to table 2 . 
Table 2. The thermal conductivity $k$ for the silicon, obtained from Eq. (1).

\begin{tabular}{|c|c|c|c|c|}
\hline$T(K)$ & $k(W / m K)$ & & $T(K)$ & $k(W / m K)$ \\
\hline 200 & 265.1 & 3 & 500 & 77.1 \\
\hline 250 & 195.3 & & 600 & 60.6 \\
\hline 300 & 152.5 & 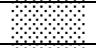 & 800 & 41.6 \\
\hline 350 & 123.9 & & 1000 & 31.2 \\
\hline 400 & 103.7 & & 1200 & 24.7 \\
\hline
\end{tabular}

Let us consider the well known steady-state conduction heat transfer problem with convective boundary condition, given by (Gama et al., 2013)

$$
\begin{gathered}
\operatorname{div}[k \operatorname{grad} T]+\dot{q}=0 \quad \text { in } \quad \Omega \\
-k \operatorname{grad} T \cdot \mathbf{n}=h\left(T-T_{\infty}\right) \quad \text { on } \quad \partial \Omega
\end{gathered}
$$

in which $\Omega$ is a bounded open set with boundary $\partial \Omega, \dot{q}$ represents an internal heat source (a known function), $h$ is the convection heat transfer coefficient, $T_{\infty}$ is the temperature of the environment (a known constant) and $\mathbf{n}$ is the unit outward normal. In Eq.(2), $T$ is the unknown and the conductivity $k$ depends on $T$.

At this point a question arises: is it important to take into account the dependence between $k$ and $T$ ?

\section{THE KIRCHHOFF TRANSFORMATION}

The Kirchhoff transformation of $T$ may be defined as follows (Arpaci, 1966)

$$
\omega=\mathrm{K}[T]=\int_{0}^{T} \hat{k}(\xi) d \xi
$$

The inverse of the Kirchhoff transformation is denoted by $\mathrm{K}^{-1}[\omega]$ and represents the temperature. In other words, $T=\mathrm{K}^{-1}[\omega]$.

Since equation (3) requires the knowledge of $k$ for any $T \in(0, \infty)$, we consider the following relations

$$
k= \begin{cases}\left\{\frac{1220}{\sqrt{T}(\ln T)}\right\}^{2}, & \text { for } \quad T \geq 200 \\ \left\{\frac{1220}{\sqrt{200}(\ln 200)}\right\}^{2}, & \text { for } T<200\end{cases}
$$

Taking into account Eq. (3), we may write

$$
\begin{aligned}
& \operatorname{grad} \omega=k \operatorname{grad} T \quad \text { and } \\
& \operatorname{div}(k \operatorname{grad} T)=\operatorname{div}(\operatorname{grad} \omega)
\end{aligned}
$$

and rewrite Eq. (2) as follows

$$
\begin{gathered}
\operatorname{div}[\operatorname{grad} \omega]+\dot{q}=0 \quad \text { in } \quad \Omega \\
-\operatorname{grad} \omega \cdot \mathbf{n}=h\left(\mathrm{~K}^{-1}[\omega]-T_{\infty}\right) \quad \text { on } \quad \partial \Omega
\end{gathered}
$$

The Kirchhoff transformation is, therefore, given by

$$
\begin{aligned}
& \omega=\mathrm{K}[T]=\int_{0}^{T} \hat{k}(\xi) d \xi=\frac{T}{200}\left\{\frac{1220}{(\ln 200)}\right\}^{2}, \\
& \quad \text { for } 0 K<T<200 K
\end{aligned}
$$

and by

$$
\begin{aligned}
\omega=\mathrm{K}[T]=\int_{0}^{200} \hat{k}(\xi) d \xi+\int_{200}^{T} \hat{k}(\xi) d \xi= \\
=\left\{\frac{1220}{(\ln 200)}\right\}^{2}+(1220)^{2} \int_{200}^{T} \frac{1}{\xi(\ln \xi)^{2}} d \xi= \\
=(1220)^{2}\left[\frac{1}{\ln 200}+\left(\frac{1}{\ln 200}\right)^{2}-\frac{1}{\ln T}\right], \\
\quad \text { for } 200 K \leq T \leq 1200 K
\end{aligned}
$$

\section{THE INVERSE OF THE KIRCHHOFF}

\section{TRANSFORMATION}

From Eqs. (3) and (4) we have that the inverse of the Kirchhoff transformation is given by

$$
\begin{aligned}
& T=\mathrm{K}^{-1}[\omega]=\omega\left(\frac{200(\ln 200)^{2}}{(1220)^{2}}\right), \\
& \text { for } \omega<\left(\frac{1220}{\ln (200)}\right)^{2} \\
& T=\mathrm{K}^{-1}[\omega]=\exp \left[\left(\frac{1}{\ln 200}+\left(\frac{1}{\ln 200}\right)^{2}-\frac{\omega}{(1220)^{2}}\right)^{-1}\right], \\
& \text { for } \omega \geq\left(\frac{1220}{\ln (200)}\right)^{2}
\end{aligned}
$$

\section{AN EXAMPLE}

Let us suppose a body represented by the set (sphere with radius $R_{2}$ )

$$
\Omega \equiv\left\{(x, y, z) \in \mathbb{R}^{3} \quad \text { such that } \quad x^{2}+y^{2}+z^{2}<R_{2}^{2}\right\}
$$

where the region $x^{2}+y^{2}+z^{2}<R_{1}^{2}$ have an uniform heat dissipation given by $Q$. 


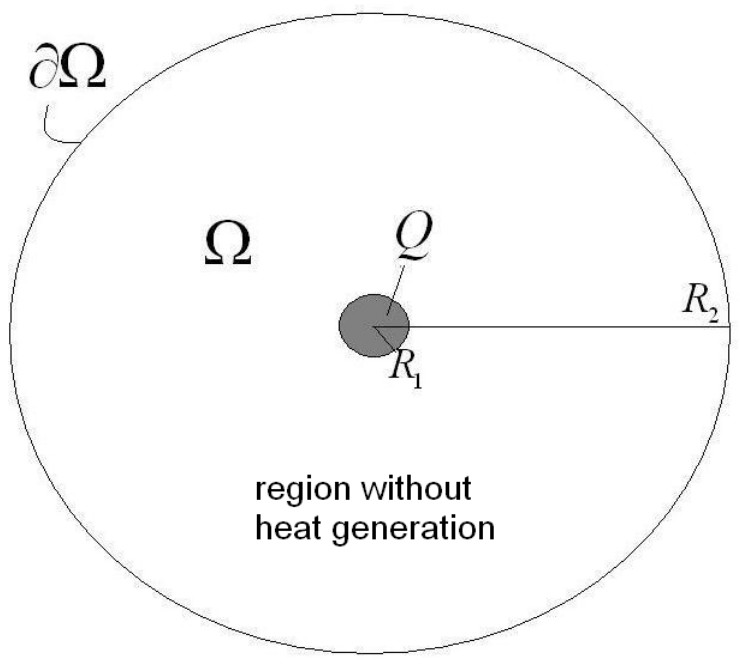

Figure 1. The spherical body.

Representing the problem in a convenient spherical coordinates system, in which $r^{2}=x^{2}+y^{2}+z^{2}$, we have

$$
\begin{aligned}
& \frac{1}{r^{2}} \frac{d}{d r}\left(r^{2} k \frac{d T}{d r}\right)+\dot{q}=0, \quad 0<r<R_{2} \\
& -k \frac{d T}{d r}=h\left(T-T_{\infty}\right), \quad r=R_{2} \\
& \text { with } \dot{q}=\left\{\begin{array}{ccc}
\frac{3 Q}{4 \pi R_{1}^{3}} & \text { for } & 0 \leq r<R_{1} \\
0 & \text { for } & R_{1} \leq r<R_{2}
\end{array}\right.
\end{aligned}
$$

or, with the aid of the Kirchhoff transformation $\omega=\mathrm{K}[T]$,

$$
\begin{aligned}
& \frac{1}{r^{2}} \frac{d}{d r}\left(r^{2} \frac{d \omega}{d r}\right)+\dot{q}=0, \quad 0<r<R_{2} \\
& -\frac{d \omega}{d r}=h\left(\mathrm{~K}^{-1}[\omega]-T_{\infty}\right), \quad r=R_{2} \\
& \text { with } \dot{q}=\left\{\begin{array}{ccc}
\frac{3 Q}{4 \pi R_{1}^{3}} & \text { for } & 0 \leq r<R_{1} \\
0 & \text { for } & R_{1} \leq r<R_{2}
\end{array}\right.
\end{aligned}
$$

The above problem has the following solution

$$
\begin{aligned}
& \omega=\frac{\dot{q}\left(R_{1}^{2}-r^{2}\right)}{6}-\frac{\dot{q} R_{1}^{3}}{3}\left(\frac{1}{R_{2}}-\frac{1}{R_{1}}\right)+ \\
& +\mathrm{K}\left[\frac{\dot{q} R_{1}^{3}}{3 h R_{2}^{2}}+T_{\infty}\right], \quad \text { for } \quad 0 \leq r<R_{1} \\
& \omega=\frac{\dot{q} R_{1}^{3}}{3}\left(\frac{1}{r}-\frac{1}{R_{2}}\right)+\mathrm{K}\left[\frac{\dot{q} R_{1}^{3}}{3 h R_{2}^{2}}+T_{\infty}\right], \\
& \quad \text { for } \quad R_{1} \leq r \leq R_{2}
\end{aligned}
$$

or,

$$
\omega=\left\{\begin{array}{l}
\frac{Q\left(R_{1}^{2}-r^{2}\right)}{8 \pi R_{1}^{3}}-\frac{Q}{4 \pi}\left(\frac{1}{R_{2}}-\frac{1}{R_{1}}\right)+ \\
+\mathrm{K}\left[\frac{Q}{4 \pi h R_{2}^{2}}+T_{\infty}\right] \text { for } 0 \leq r<R_{1} \\
\frac{Q}{4 \pi}\left(\frac{1}{r}-\frac{1}{R_{2}}\right)+\mathrm{K}\left[\frac{Q}{4 \pi h R_{2}^{2}}+T_{\infty}\right] \\
\text { for } R_{1} \leq r \leq R_{2}
\end{array}\right.
$$

with

$$
T=\left\{\begin{array}{l}
\mathrm{K}^{-1}\left\{\frac{Q\left(R_{1}^{2}-r^{2}\right)}{8 \pi R_{1}^{3}}-\frac{Q}{4 \pi}\left(\frac{1}{R_{2}}-\frac{1}{R_{1}}\right)+\right. \\
\left.+\mathrm{K}\left[\frac{Q}{4 \pi h R_{2}^{2}}+T_{\infty}\right]\right\}, \quad \text { for } 0 \leq r<R_{1} \\
\mathrm{~K}^{-1}\left[\frac{Q}{4 \pi}\left(\frac{1}{r}-\frac{1}{R_{2}}\right)+\mathrm{K}\left[\frac{Q}{4 \pi h R_{2}^{2}}+T_{\infty}\right]\right], \\
\quad \text { for } \quad R_{1} \leq r \leq R_{2}
\end{array}\right.
$$

It is easy to note that the temperature at $r=R_{2}$ does not depend on the thermal conductivity, and is given by

$$
\left.T\right|_{r=R_{2}}=\frac{Q}{4 \pi h R_{2}^{2}}+T_{\infty}
$$

Now, let us evaluate the temperature at $r=R_{1}$ considering two cases:

Case 1): $k$ given by Eq. (4)

Case 2): $k=k_{0}=$ constant , in which $k_{0}$ is the mean value of $k$ obtained from the above case for $R_{1} \leq r \leq R_{2}$.

In order to provide explicit results, let us consider the following data:

$$
\begin{aligned}
& Q=300 \pi W, \quad \frac{Q}{4 \pi h R_{2}^{2}}+T_{\infty}=200 K, \\
& R_{1}=0.001 m, \quad R_{2}=0.005 m
\end{aligned}
$$

Thus, for case 1 , we have

$$
\begin{aligned}
& \omega=75\left(\frac{1}{r}-\frac{1}{0.005}\right)+\left(\frac{1220}{\ln 200}\right)^{2}, \\
& \text { for } \quad 0.001 \leq r \leq 0.005
\end{aligned}
$$


and

$$
\begin{aligned}
& T=\mathrm{K}^{-1}\left[75\left(\frac{1}{r}-\frac{1}{0.005}\right)+\left(\frac{1220}{\ln 200}\right)^{2}\right] \\
& \text { for } 0.001 \leq r \leq 0.005
\end{aligned}
$$

So, once that $T \geq 200 K$ everywhere, we have

$$
\begin{aligned}
& T=\mathrm{K}^{-1}[\omega]= \\
& =\exp \left[\left(\frac{1}{\ln 200}+\left(\frac{1}{\ln 200}\right)^{2}-\frac{\omega}{(1220)^{2}}\right)^{-1}\right]
\end{aligned}
$$

with $\omega$ given by (17).

Hence, at $r=R_{1}=0.001 \mathrm{~m}$ the temperature is $T=843.2 \mathrm{~K}$.

The mean value of $k$ for $200 K<T<843.2 K$ is $152.0 \mathrm{~W} / \mathrm{mK}$. Now, let us consider the case 2 with $k=k_{0}=152 \mathrm{~W} / \mathrm{mK}$.

The expression for $\omega$ is the same one. However, $T$ is given by

$$
\begin{aligned}
& T=\frac{\omega}{k_{0}}=\frac{1}{152}\left\{\frac{Q}{4 \pi}\left(\frac{1}{r}-\frac{1}{R_{2}}\right)+\right. \\
& \left.+(152)\left(\frac{Q}{4 \pi h R_{2}^{2}}+T_{\infty}\right)\right\}, \quad \text { for } \quad R_{1} \leq r \leq R_{2}
\end{aligned}
$$

Inserting the considered data, Eq. (19) becomes

$$
\begin{aligned}
& T=\frac{75}{152}\left(\frac{1}{r}-\frac{1}{0.005}\right)+200, \\
& \quad \text { for } \quad 0.001 \leq r \leq 0.005
\end{aligned}
$$

So, at $r=R_{1}=0.001 \mathrm{~m}$ we have $T=594.7 \mathrm{~K}$. In other words, the constant conductivity assumption gives rise to a temperature $248.5 \mathrm{~K}$ below the actual one. If the conductivity was assumed the one for $T=340 K \quad(k=130 \mathrm{~W} / \mathrm{mK})$, the temperature at $r=R_{1}=0.001 \mathrm{~m}$ would be equal to $T=661.5 \mathrm{~K}$.

\section{FINAL REMARKS}

In this work it was shown that the dependence between the thermal conductivity and the temperature can not be neglected without a careful analysis. When this analysis is not possible before the simulation, the obtained results may give an insight about how much they are reliable. For instance, after a simulation assuming constant thermal conductivity, we must verify if the temperature levels are compatible with the considered value for the thermal conductivity.

\section{ACKNOWLEDGEMENTS}

The author gratefully acknowledge the financial support provided by the Brazilian agency $\mathrm{CNPq}$.

\section{REFERENCES}

Arpaci, V. S., 1966, Conduction Heat Transfer, Addison-Wesley Publishing Company, Inc..

Gama, R. M. S., Corrêa, E. D., and MartinsCosta M. L., 2013, An Upper Bound Estimate for the Steady-state Temperature for a Class of Heat Conduction Problems wherein the Thermal Conductivity is Temperature Dependent, International Journal of Engineering Science, Vol. 69, pp. 77-83.

Glassbrenner, C. J., and Slack, G. A., 1964, Thermal Conductivity of Silicon and Germanium from $3^{\circ} \mathrm{K}$ to the Melting Point- Phys. Rev, Vol. 134, pp. 1058-1069.

Goldberg Yu., Levinshtein M. E., and Rumyantsev, S. L., 2001, Properties of Advanced Semiconductor Materials GaN, AlN, SiC, BN, SiC, SiGe, John Wiley \& Sons, Inc..

Incropera, F., and Dewitt, P. D., 1996, Introduction to Heat Transfer, 3rd edition, John Wiley\&Sons Inc.. 